\title{
Amisulprida una nueva profilaxis contra las náuseas y vómitos postoperatorios: una revisión de la literatura
}

\author{
Amisulpride new prophylaxis against postoperative nausea and vomiting: a review of the literature \\ Cecilia Gonzalez ${ }^{1}$, Maria Jesus Garchitorena' ${ }^{1}$ Karime Sabat ${ }^{1}$
}

\section{Resumen:}

Introducción: las náuseas y vómitos postoperatorios (PONV) son una complicación postoperatoria común de la anestesia, por lo que la búsqueda de nuevas profilaxis efectivas ha aumentado durante los últimos años, en este contexto se perfila como una opción efectiva el amisulprida, antipsicótico que actúa bloqueando los receptores dopaminérgicos D2 y D3, en dosis bajas. Métodos: se realizó una búsqueda en bases de datos establecidas como: Pubmed, Embase, Google Scholar, Medline y la Cochrane central registed of controlled trials. Con las siguientes definiciones clave: "NV" or "postoperative nausea and vomiting" or "postoperative nausea" or "postoperative vomiting" and "Amisulpride" or "APD421", con el fin de cumplir el objetivo general de verificar la efectividad de amisulprida como medicamento profiláctico en manejo de PONV. Resultados: se evaluaron 3 estudios multicéntricos randomizados controlados, con alto grado de confiabilidad y buena calidad metodológica. Se evidencia que el amisulprida es un medicamento profiláctico efectivo contra PONV y con buen perfil de seguridad. Discusión/Conclusión: el amisulprida es un medicamento profiláctico eficaz para prevención de PONV, en dosis óptima de $5 \mathrm{mg}$, administrado durante el intraoperatorio de cirugías electivas en que se utilice anestesia general en pacientes de moderado a alto riesgo de PONV según escala de Apfel.

Palabras clave: amisulprida; APD421; náuseas postoperatorias; vómitos postoperatorios; náuseas y vómitos postoperatorios; profilaxis.

\begin{abstract}
Introduction: postoperative nausea and vomiting (PONV) are common postoperative complications of anaesthesia, that is why the search for new effective prophylaxis has increased in recent years. Between the drugs that have been tested for this purpose, Amisulpride, an antipsychotic that blocks D2 and D3 dopaminergic receptors, seems to be an effective option when used in low doses. Methods: This search was done by using databases such as Pubmed, Embase, Google Scholar, Medline and the Cochrane central register of controlled. With the following keywords: "NV" or "postoperative nausea and vomiting" or "postoperative nausea" or "postoperative vomiting" and "Amisulpride" or "APD421", with the purpose of verifying the effectiveness of Amisulpride as prophylactic medication in PONV management, which is our main objective. Results: 3 randomised, controlled, multicenter, with high reliability and good methodological quality studies were evaluated. Evidence suggests that Amisulpride is effective as a prophylactic medication against PONV, and it also has a good safety profile. Discussion/Conclusion: Amisulpride administered in an optimal dose of 5 mg during intraoperative elective surgeries that required general anaesthesia, showed to be effective in preventing PONV as a prophylactic drug in patients with moderate to high risk of PONV according to the Apfel scale.
\end{abstract}

Keywords: Amisulpride; APD421; Postoperative nausea; Postoperative vomiting; Prophylaxis.

Fecha de envío: 2020-07-24 - Fecha de aceptación: 2020-12-10

\section{Introducción}

Las náuseas y vómitos postoperatorios (PONV) son un problema frecuente después de someterse a una cirugía, siendo la principal causa de hospitalización en cirugía ambulatoria (Hill et al., 2000). La incidencia de estos eventos sin profilaxis para náuseas y vómitos postoperatorios varía entre un 20 a un $30 \%$ en cirugías con uso de anestesia general

(1) Facultad de Medicina, Pontificia Universidad Católica de Chile

Autor de correspondencia:Cigonzalez5@uc.cl 
(Veiga-Gil et al., 2017), incrementando los costos en la hospitalización por uso de fármacos antieméticos, mayor sobrecarga al personal de salud, incomodidad en los pacientes y frecuencia de eventos adversos asociados a los vómitos como procesos aspirativos, alteraciones electrolíticas y deshidratación (Lagos et al., 2009).

Está bien establecido que la etiología de PONV es multifactorial, confluyendo factores del paciente, de la anestesia y de la cirugía misma (Lagos et al., 2009). Los factores de riesgo se relacionan y establecen un riesgo estratificado por diferentes escalas, siendo la más utilizada en población adulta el score simplificado de Apfel que divide a los pacientes en bajo, bajo a moderado, alto y extremadamente alto riesgo de PONV, este último alcanzando un $80 \%$ de riesgo (Pierre et al., 2002).

Lo primordial en esta materia es la prevención de PONV, para lo cual se requiere un abordaje multifactorial según la evaluación preoperatoria del riesgo (Gómez-Arnau et al., 2011). Dentro de la estrategia farmacológica en la mayoría se requiere profilaxis combinada con diferentes antieméticos, pese a esto ninguno de los fármacos actuales ofrece protección completa, la reducción del riesgo con monodroga es de sólo un $26 \%$ y, al combinarse fármacos bajo la estrategia multimodal, se alcanza una reducción máxima de hasta un 56\% (Apfel et al., 2004), de aquí deriva la búsqueda de nuevos fármacos útiles en este ámbito.

Los fármacos utilizados como profilaxis para PONV son los antagonistas de los receptores 5-HT3, D2 y H1, corticoides y anticolinérgicos. Siendo los más frecuentemente utilizados en la práctica clínica: ondansetrón, corticoides (dexametasona) y el droperidol (ASPAN'S et al., 2006). A comienzos del siglo XXI el droperidol, fármaco antagonista del receptor D2 se configuró como un gran agente en la prevención de PONV con altas tasas de reducción de estos eventos postoperatorios, sin embargo, su uso se descontinuó en la mayoría de las partes por una alerta de la FDA de alto riesgo de prolongación del intervalo QT y síndrome extrapiramidal (Habib \& Gan, 2008). Es por esto, que en los últimos años en la búsqueda de nuevos agentes profilácticos de PONV se comienza a estudiar la eficacia del amisulprida en la profilaxis de PONV, fármaco antagonista del receptor dopaminérgico D2 y D3, utilizado frecuentemente en el ámbito de la psiquiatría como antisicótico en dosis de 50 a 1200 $\mathrm{mg}$ /día que lo configuran como un fármaco seguro y con buena tolerabilidad incluso en uso crónico (Juruena \& De Oliveira, 2010). La fisiología del formato endovenoso del amisulprida creado para la profilaxis de PONV, cuyo nombre es APD421, es el bloqueo de los receptores D2 y D3 de dopamina con lo cual inhibe la capacidad de los opioides de producir emesis y náuseas al estimular la zona gatillo quimiorreceptora, que a su vez activa indirectamente al centro del vómito (León-Alvarez, 2013).
Esta revisión tiene por objetivo definir la eficacia y seguridad del amisulprida intravenoso en la profilaxis de náuseas y vómitos postoperatorios en dosis únicas bajas en pacientes mayores de 18 años sometidos a cirugías electiva con uso de anestesia general y que posean moderado a alto riesgo de PONV según criterios Apfel.

\section{Métodos}

1. Criterios de selección: los estudios fueron escogidos según el cumplimiento de los criterios de selección previamente establecidos que corresponden a: (1) población de estudio: adultos mayores de 18 años que reciben anestesia general en cirugía electiva tanto abierta como laparoscópica y que presentan 2 o más puntos de la escala Apfel que determina riesgo de PONV. (2) Intervención: administración de amisulprida como profilaxis de PONV una hora posterior a inducción de anestesia general.(3) Comparación: versus placebo. (4) Objetivos: determinar incidencia de respuesta completa de amisulprida profiláctico, definido como no tener episodio de náuseas o vómitos en tiempo postoperatorio en un máximo de 72 horas, determinar severidad de síntomas con escala verbal de 0-11 puntos de medición de náuseas y vómitos. (5) Tipo de estudio: se seleccionaron estudios randomizados controlados, doble ciego multicéntricos. (6) Criterios de exclusión: abstract publicados sin acceso a artículo completo, estudios que no presentaban objetivos determinados como outcome primarios, artículos con objetivos poco claros o no coincidente con los seleccionados.

2. Fuentes de información y búsqueda: se buscaron estudios randomizados controlados que determinaran la utilidad de amisulprida como medicamento profiláctico en PONV con un perfil de seguridad óptimo. Se realizó la búsqueda en bases de datos: Pubmed, Embase, Google Scholar, Medline y la Cochrane central registed of controlled trials. Con las siguientes definiciones clave: "NV" or "postoperative nausea and vomiting" or "postoperative nausea" or"postoperative vomiting" and "Amisulpride" or "APD421".

3. Selección deestudiosy recolección de datos: de los tres investigadores, dos registraron la información de cada estudio por separado, en la evaluación de riesgo de sesgo y resultados. Cuando se mostraron discrepancias, el tercer investigador determinó el consenso final.

4. Objetivos: El objetivo general corresponde a verificar la efectividad de amisulprida como medicamento profiláctico en manejo de PONV, esto definido como respuesta completa (no tener episodios de náuseas ni vómitos postoperatorios, ni uso de antieméticos de resctate en un máximo de 72 horas).

Los objetivos específicos corresponden a: evaluar la eficacia de las diferentes dosis de amisulprida versus placebo en manejo de PONV. Analizar el perfil de seguridad del amisulprida versus placebo. 


\section{Resultados}

1. Característica de estudios: de la búsqueda inicial resultaron 20 estudios, de los cuales se seleccionaron 7 en base a su abstract, los que corresponden a artículos completos y disponibles, de los analizados escogimos finalmente 3 RCTs que cumplían con todos los criterios de selección. Además, los estudios seleccionados se encuentran publicados en revistas de alto impacto y se encuentran debidamente inscritos en Clinicaltrials.gov, lo que otorga mayor confiabilidad. Figura1

2. Riesgo de sesgo: el nivel de confiabilidad de los estudios evaluados resultó ser alto, estos fueron evaluados con el manual de determinación de sesgo de la revista Cochrane. Sólo se evidenció riesgo de sesgo indeterminado en dos items, que a nuestra evaluación no presenta influencia en el modelo y resultado de los estudios. Tabla 1.

3. Eficacia: respecto a lo revisado en la literatura, determinamos que el amisulprida en cuanto a su respuesta completa, como profilaxis contra PONV, muestra ser significativamente efectivo versus placebo, en los tres estudios evaluados. Los estudios, además, presentan alto grado de confiabilidad, ya que solo se escogieron RCTs, multicéntricos, los cuales fueron evaluados según el sistema GRADE, lo cual se evidencia en la tabla 2.

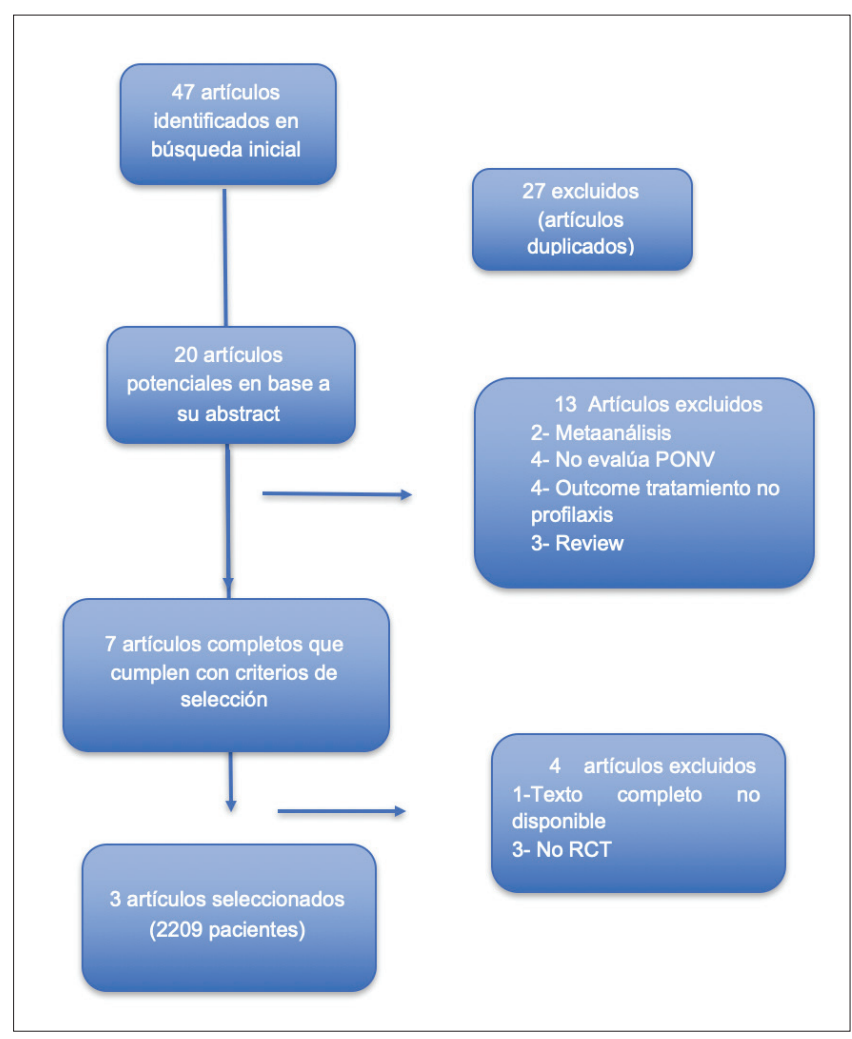

Figura 1: Selección de estudios. diagrama de flujo que evidencia proceso de selección de estudios según criterios de inclusión.

Tabla 1: Evaluación sesgo. Tabla muestra riesgo de sesgo individual según categoría, de cada estudio escogido para la revisión.

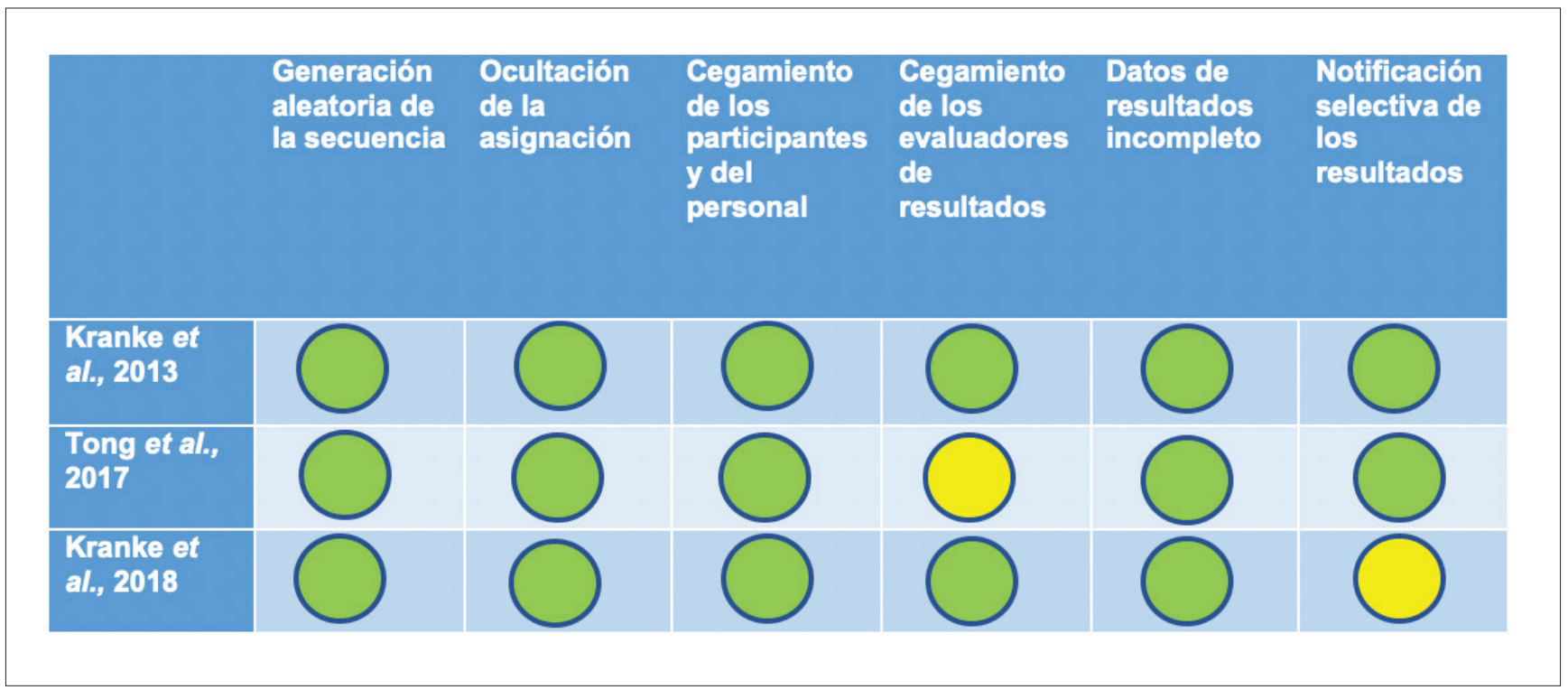


Tabla 2: Análisis GRADE. Se caracterizan los tres estudios incluidos en revisión bibliográfica para estimar su nivel de confiabilidad.

\begin{tabular}{|c|c|c|c|c|c|c|c|c|}
\hline Estudio & País & Participantes & $\begin{array}{l}\text { Pacientes } \\
\text { (N) }\end{array}$ & $\begin{array}{l}\begin{array}{l}\text { Tipo } \\
\text { estudio }\end{array} \\
\end{array}$ & Tipo cirugía & Anestesia & Grupos & Objetivos \\
\hline $\begin{array}{l}\text { Kranke } \\
\text { et } \\
\text { al.,2013 }\end{array}$ & $\begin{array}{l}\text { Alemania, } \\
\text { Francia y } \\
\text { USA }\end{array}$ & $\begin{array}{l}\text { Adultos } \\
\text { mayores de } \\
18 \text { años } \\
2 \text { o más } \\
\text { puntos en } \\
\text { escala Apfel }\end{array}$ & 215 & RCT & $\begin{array}{l}\text { Cirugía } \\
\text { electiva } \\
\text { abierta o } \\
\text { laparoscópica }\end{array}$ & $\begin{array}{l}\text { Anestesia } \\
\text { inhalatoria } \\
\text { Mayor a } 1 \\
\text { hora } \\
11 \text { VRS }\end{array}$ & $\begin{array}{ll}\text { 1. Amisulprida } \\
1 \mathrm{mg}(58) \\
\text { 2. Amisulprida } \\
5 \mathrm{mg}(50) \\
\text { 3. Amisulprida } \\
20 \mathrm{mg}(53) \\
\text { 4. Placebo } \\
\\
\text { (54) }\end{array}$ & $\begin{array}{l}\text { Primario: incidencia de } \\
\text { NVPO } \\
\text { Secundario: } \\
\text { Incidencia de náuseas } \\
\text { severas, severidad de } \\
\text { náuseas }\end{array}$ \\
\hline $\begin{array}{l}\text { Tong et } \\
\text { al., } 2017\end{array}$ & $\begin{array}{l}\text { Alemania } \\
\text { y Francia }\end{array}$ & $\begin{array}{l}\text { Adultos } \\
\text { mayores de } \\
18 \text { años } \\
2 \text { o más } \\
\text { puntos en } \\
\text { escala Apfel }\end{array}$ & 689 & RCT & $\begin{array}{l}\text { Cirugía } \\
\text { electiva }\end{array}$ & $\begin{array}{l}\text { Anestesia } \\
\text { general } \\
\text { Mayor a } 1 \\
\text { hora }\end{array}$ & $\begin{array}{ll}\text { 1. Amisulprida } \\
5 \mathrm{mg}(345) \\
\text { 2. Placebo } \\
\\
(344)\end{array}$ & $\begin{array}{l}\text { Primario: } \\
\text { Incidencia de respuesta } \\
\text { completa de amisulprida } \\
\text { Secundario: } \\
\text { Incidencia de eventos } \\
\text { aislados de náuseas o } \\
\text { vómitos }\end{array}$ \\
\hline $\begin{array}{l}\text { Kranke } \\
\text { et al., } \\
2018\end{array}$ & Alemania & $\begin{array}{l}\text { Adultos } \\
\text { mayores de } \\
18 \text { años } \\
3 \text { o más } \\
\text { puntos en } \\
\text { escala Apfel }\end{array}$ & 1147 & RCT & $\begin{array}{l}\text { Cirugía } \\
\text { electiva } \\
\text { abierta o } \\
\text { laparoscópica }\end{array}$ & $\begin{array}{l}\text { Anestesia } \\
\text { general o } \\
\text { inhalatoria } \\
\text { mayor a } 1 \\
\text { hora } \\
11 \text { VRS }\end{array}$ & $\begin{array}{ll}\text { 1. } & \text { Amisulprida } \\
& 5 \mathrm{mg}(572) \\
& + \\
& \text { antiemético } \\
& \text { usual } \\
\text { 2. } & \text { Placebo } \\
& (575)+ \\
& \text { antiemético } \\
& \text { usual }\end{array}$ & $\begin{array}{l}\text { Primario: } \\
\text { Incidencia de respuesta } \\
\text { completa de amisulprida } \\
\text { Secundario: } \\
\text { Incidencia de náuseas o } \\
\text { vómitos }\end{array}$ \\
\hline
\end{tabular}

En cuanto a los resultados por estudio, se evidencia que hay una incidencia de respuesta completa de 10 a 12\% (según el centro evaluado) de amisulprida en dosis de $5 \mathrm{mg}$ versus placebo y una disminución en la incidencia de PONV de hasta un 20\% (Tong et al., 2017).

Por su parte el equipo de Kranke en el 2013 describen una disminución en la incidencia de PONV de hasta un 29\% (grupo 5mg) y de riesgo relativo de hasta un 42\%, además evidencian que el grupo de amisulprida $5 \mathrm{mg}$ es significativamente superior en efectividad al grupo que se le administró 1 mg, sin embargo, al comparar amisulprida $5 \mathrm{mg}$ con dosis superiores de $20 \mathrm{mg}$, demostró que la dosis más efectiva era $5 \mathrm{mg}$, ya que en este último se evidenció una incidencia de $40 \%$ de PONV versus un $57 \%$ en el grupo de 20 mg de amisulprida (Kranke et al., 2013). Por su parte, al administrar amisulprida en dosis de $5 \mathrm{mg}$ asociado a un antiemético estándar, se evidenció una incidencia de respuesta completa de $11 \%$ mayor que el grupo placebo que solo recibió el mismo antiemético estándar, como prevención de PONV en pacientes con riesgo alto de complicación según escala Apfel y una reducción del riesgo relativo de hasta un $20 \%$ (Kranke et al., 2018). Por otra parte, se evaluó la incidencia de náuseas y vómitos como episodios aislados, demostrando también una disminución significativa de estos eventos (8 a 10\% menos en grupo amisulprida) (Tong et al., 2017; Kranke et al.,2018).

Perfil de seguridad amisulprida: en los tres estudios evaluados se consideró un perfil óptimo de seguridad del amisulprida versus placebo. No se evidenciaron alteraciones significativas en parámetros hemodinámicos, ni electrocardiográficos en ninguno de los tres estudios. En uno de los estudios se evalúa los niveles de prolactina, no evidenciándose hiperprolactinemia asociada al uso de amisulprida (Tong et al., 2017). En cuanto a eventos graves y mortalidad, se descarta en los tres estudios que haya sido asociado a uso de amisulprida. La causalidad de estos eventos se definió como complicaciones de la cirugía.

\section{Discusión}

De la revisión de trabajos realizada, se puede desprender en forma global que el uso de amisulprida como profilaxis de PONV sería una práctica segura y con evidencia acerca de su utilidad. Dado la calidad de los estudios seleccionados, en cuanto a su diseño, metodología y capacidad de ser reproducidos, poseer una muestra representativa y grande de pacientes, cuyas características físicas se distribuyeron homogéneamente con respecto al grupo control, confiamos en la solidez de este análisis para propiciar un cambio de conducta clínico.

En el estudio realizado por Kranke en el 2013 se evidencia que el uso de amisulprida en forma endovenosa previo al inicio de la cirugía es seguro y efectivo en reducir PONV en las primeras 24 horas postoperatorias, en un grupo de pacientes adultos de riesgo alto a moderado de sufrir PONV, determinando como dosis óptima $5 \mathrm{mg}$ (Kranke et al., 2013). Se determina esta dosis ya que demostró ser superior en disminuir la incidencia de PONV que el uso de dosis menores de $1 \mathrm{mg}$ o incluso placebo, también demostró ser más efectiva que la dosis de $20 \mathrm{mg}$. 
La incidencia de PONV en el grupo placebo de la población estudiada por Kranke en el 2013 es de un 69\% lo cual es consistente con lo reportado en la literatura en pacientes de moderado a alto riesgo de PONV lo cual brinda confiabilidad y nos permite deducir que el beneficio evidenciado al uso de amisulprida aplica en esta población (Kranke et al., 2013). De la misma forma, el grupo placebo de la población estudiada por Tong en el 2017 evidenció una incidencia de PONV de 59,5\% (Tong et al., 2017).

Para determinar la utilidad en el uso de amisulprida, debemos considerar comparar la efectividad de amisulprida con otros antieméticos de uso actual. Un metanálisis de Cochrane que incluyó 737 estudios, y en total 103237 pacientes, consideró 8 medicamentos utilizados como buenos antieméticos (droperidol, metoclopramida, ondansetron, tropisetron, dolasetron, dexametasona, cyclizina y granisetron) y determinaron que en general, actúan con un rango de reducción de riesgo relativo de PONV entre un 20-40\% (Carlisle \& Stevenson, 2006). Por otra parte, en este estudio realizado por Kranke en el 2013, se determinó una reducción de riesgo relativo por parte del uso de amisulprida de 42\% (Kranke et al., 2013), lo que parece prometedor al ser comparado con lo que se utiliza en la práctica habitual. Otro punto a destacar en este estudio es la aparición de efectos no deseados por el uso de antieméticos, en éste se determina que amisulprida no pareciera ser cardiotóxico, ya que no hubo diferencias en parámetros electrocardiográficos ni de intervalo QT corregido entre los grupos del estudio, ni a 1 hora ni a las 24 horas postoperatorias. No obstante, son prudentes al transparentar que los electrocardiogramas registrados de sus pacientes no permiten descartar por completo alteraciones en el intervalo QT, esto ya que podrían no ser lo suficientemente detallados. Por otra parte, efectos como la cefalea, consideran que no son atribuibles al uso de amisulprida en dosis de $5 \mathrm{mg}$ endovenoso (Kranke et al., 2013).

La efectividad demostrada en ese entonces, concuerda con lo replicado más tarde por Tong en el 2017 donde se analizan dos centros en los que se demuestra su utilidad versus placebo en reducir hasta un $20 \%$ el riesgo relativo de PONV (Tong et al., 2017). Es relevante destacar que amisulprida en estos estudios demostró que la incidencia de efectos adversos asociados a tratamiento parece ser levemente mayor al grupo que utilizó placebo, aumentando en no más de 1 a $2 \%$. Incluso presentando menor evidencia de hipotensión, hiperglicemias y anemia postoperatoria el grupo que recibió amisulprida.

Estos dos estudios mencionados incluyeron pacientes con 2 o más factores de riesgo para PONV, sin embargo, en nuestro tercer estudio seleccionado, realizado el 2018 por Kranke se acotó la muestra a pacientes con 3-4 factores de riesgo, es decir quienes se consideran como de alto riesgo. Lo particular de esto es que en este estudio se vio la utilidad de amisulprida al ser administrado junto a otro antiemético profiláctico (ondansetrón o dexametasona) ya que estos pacientes tienen indicación de profilaxis combinada con fármacos que actúen en diferentes receptores. Aquí se destaca que al añadir amisulprida a la profilaxis de PONV al momento de la inducción anestésica, en este grupo de alto riesgo, se obtiene hasta un $11 \%$ más de respuesta versus el grupo control, es decir reduce la necesidad de medicamentos de rescate e incidencia de vómitos en las 24 horas postoperatorias y una reducción de riesgo relativo de PONV de hasta un 20\% (Kranke et al., 2018). La ventaja de amisulprida es que puede utilizarse en conjunto con otros fármacos, ya que, dado su farmacocinética, no es inhibidor ni sustrato del citocromo P450 (Mora et al., 2015).

Por esto, no sólo sería útil en prevenir PONV en un grupo de pacientes de riesgo moderado- alto, sino que además en pacientes que requieren una profilaxis de PONV combinada se configura como una alternativa óptima y, de esta forma, reservar otra línea de antieméticos como terapéuticos, como por ejemplo el ondansetrón, ya que los medicamentos usados como profilaxis no deberían ser utilizados como tratamiento cuando se requiera.

Por otra parte, contemporáneo a la realización de esta revisión se publicó una revisión sistemática respecto a la eficacia de la amisulprida como profilaxis de PONV, que estipula que bajas dosis de este medicamento sería eficaz y seguro para prevenir las náuseas y vómitos postoperatorios (Habib et al., 2019). Además, existen estudios en desarrollo que hasta ahora muestran resultados también favorables respecto a la eficacia de la amisulprida (Zhang et al., 2020).

Dentro de las limitaciones que podemos observar en esta revisión es que uno de los estudios no especifica los efectos adversos asociados a amisulprida, lo que era en realidad un punto clave para tener conclusiones más sólidas acerca de la seguridad del fármaco, por ser el estudio con mayor tamaño muestral y además el más reciente (Kranke et al., 2018). De todas formas, se refieren a que no hubo cambios en el electrocardiograma ni signos extrapiramidales, que son uno de los efectos adversos más importantes. Otra limitación importante es el número de RCTs asociados al tópico que estamos tratando, dos de ellos realizados por el mismo autor principal en diferentes años y con equipos diferentes, ya que los estudios son recientes y el potencial uso del amisulprida como profilaxis de PONV es un tema que se está tocando recientemente en el área de anestesiología.

\section{Conclusión}

El amisulprida es un medicamento profiláctico eficaz para prevención de PONV, en dosis óptima de 5 mg, administrado durante el 
intraoperatorio de cirugías electivas en que se utilice anestesia general en pacientes de moderado a alto riesgo de PONV según escala de Apfel. La importancia de esto radica en la necesidad de utilizar nuevos fármacos antieméticos con buen perfil de seguridad y eficaces para tratamiento único y combinado que usualmente requieren estos pacientes.

El amisulprida como fármaco profiláctico de PONV, en su forma comercial Barhemsys, fue recientemente aprobado por la FDA en febrero del 2020, por lo que se espera que esté disponible en Chile durante el 2021.

\section{Reconocimientos}

Este trabajo no cuenta con fuentes de financiamiento externas y los autores declaran no poseer conflictos de interés.

\section{Referencias}

Apfel C, Korttila K, Abdalla M, Kerger H, Turan A, Vedder I, et al (2004). A factorial trial of six interventions for the prevention If postoperative nausea and vomiting. New England Journal of Medicine 350, 2441-2451.

ASPAN'S Evidence-Based Clinical Practice Guidelone for the Prevention and/or Management of PONV/PDNV (2006). Journal of PeriAnesthesia Nursing 21, 230-250.

Carlisle J. \& Stevenson C. (2006). Drugs for preventing postoperative nausea and vomiting. Cochrane Database of Systematic Reviews

Gómez-Arnau J, Aguilar J, Bovaira P, Bustos F, De Andrés J, De la pinta $J$, et al (2011). Recomendaciones de prevención y tratamiento de las náuseas y vómitos postoperatorios y asociados a las infusiones de opioides. Revista Española de Anestesiología y Reanimación 57, 508-524.

Habib A\& Gan T (2008). The use of droperidol before and after the Food and Drug Administration black box warning: a survey of the members of the Society of Ambulatory Anesthesia. Journal of Clinical Anesthesia 20, 35-39.

Habib A, Kranke P, Bergese S, Chung, F, Ayad S, Siddiqui N, Motsch J, Leiman D , Melson T, Diemunsch P, Fox G. \& Candiotti K. (2019). Amisulpride for the Rescue Treatment of Postoperative Nausea or Vomiting in Patients Failing Prophylaxis: A Randomized, Placebo-controlled Phase III Trial. Anesthesiology 130, 203-212.
Hill R, Lubarsky D, Phillips-Bute B, Fortney J, Creed M, Glass P et al (2000). Cost-effectiveness of Prophilactic Antiemetic Therapy with Ondansetron, Droperidol or Placebo. American society of Anesthesiologist 92, 958-967.

Juruena M \& De Oliveira I (2010). Safety and tolerability of antipsychotics: focus on amisulpride. Drug, Healthcare and Patient Safety 2, 205-211.

Kranke P, Bergese S, Minkowitz H, Melson T, Leiman D, Candiotti $\mathrm{K}$ et al (2018). Amisulpride Prevents Postoperative Nausea and Vomiting in Patients at High Risk. Anesthesiology 128, 1099-1106.

Kranke P, Eberhart L, Motsch J, Chassard D, Wallenborn J, Diemunsch P et al (2013). I.V. APD421 (amisulpride) prevents postoperative nausea and vomiting: a randomized, double-blind, placebo-controlled, multicentre trial. British Journal of Anaesthesia 111, 938-945.

Lagos C.\& Quezada S. (2009). Profilaxis y Tratamiento de las Náuseas y Vómitos postoperatorios. Revista Chilena de Anestesiología 38,24-33.

Leon-Álvarez E. (2020). Neurofisiología de la náusea y vómito. Revista Mexicana de Anestesiologia S361-S362.

Mora F, Molina J, Zubillaga E, López-Muñoz F \& Álamo C. (2015). CYP450 and Its Implications in the Clinical Use of Antipsychotic Drugs. Clinical \& Experimental Pharmacology 05.

Pierre S, Benais H, Pouymayou J (2002). Apfel's simplied score may favourably predict the risk of postoperative nausea and vomiting. Canadian Journal of Anesthesia/Journal canadien d'anesthésie 49, 237-24

Tong G, Kranke P, Minkowitz H, Bergese S, Motsch J (2017). Intravenous Amisulpride for the Prevention of Postoperative Nausea and Vomiting. Anesthesiology 126, 268-275.

Veiga-Gil L, Pueyo J \& López-Olaondo L (2017). Náuseas y vómitos postoperatorios: fisiopatología, factores de riesgo, profilaxis y tratamiento. Revista Española de Anestesiología y Reanimación 64, 223-232.

Zhang L, Zhang F, Tang W, He L, Liu Y, Tian D. \& Ai Y. Efficacy of amisulpride on postoperative nausea and vomiting: a systematic review and meta-analysis. Eurepean Journal Clinical Pharmacology 76, 903-912. 